\section{$\underset{\substack{\text { hommes } \\ \text { \& migrations }}}{ }$}

\section{Hommes \& migrations}

Revue française de référence sur les dynamiques

migratoires

\section{$1299 \mid 2012$}

Musulmanes et féministes en Grande-Bretagne

\title{
Femmes de culture musulmane et participation politique
}

En France et en Grande-Bretagne

\section{Khursheed Wadia}

\section{(2) OpenEdition}

Journals

Édition électronique

URL : http://journals.openedition.org/hommesmigrations/1403

DOI : 10.4000/hommesmigrations. 1403

ISSN : 2262-3353

Éditeur

Musée national de l'histoire de l'immigration

Édition imprimée

Date de publication : 1 septembre 2012

Pagination : 24-31

ISSN : $1142-852 X$

\section{Référence électronique}

Khursheed Wadia, "Femmes de culture musulmane et participation politique », Hommes \& migrations [En ligne], 1299 | 2012, mis en ligne le 31 décembre 2014, consulté le 01 mai 2019. URL : http:// journals.openedition.org/hommesmigrations/1403; DOI : 10.4000/hommesmigrations. 1403 


\section{Femmes de culture musulmane et participation politique en France et en Grande-Bretagne}

Par Khursheed Wadia, principal research fellow, université de Warwick

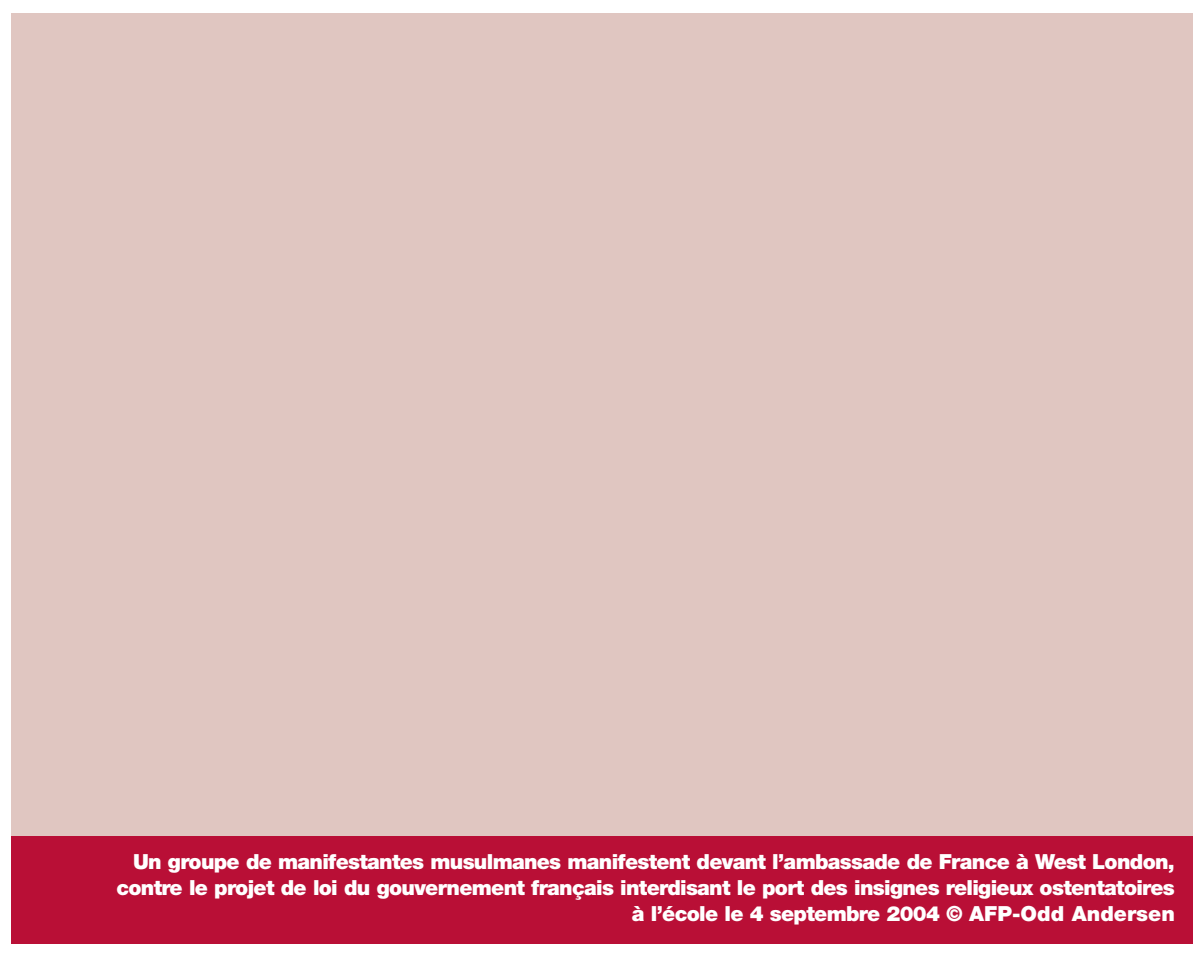

En France comme en Grande-Bretagne, la participation politique des femmes de culture musulmane souffre d'un déficit d'analyse.

Perçues comme peu éduquées, cantonnées à leur foyer, elles sont doublement exclues, en tant que femmes et musulmanes, des discours publics essentiellement portés par des hommes. Si l'intérêt de ces femmes pour la politique demeure peu interrogé, leur capacité d'engagement démontre dans les faits un profond attachement pour la chose publique. 
L'un des premiers objectifs de notre étude ${ }^{(1)}$ était de remettre en question les préjugés à l'encontre des femmes des communautés musulmanes: montrer qu'elles ne sont ni soumises, ni apathiques, ni ignorantes, mais qu'elles ont la capacité et la volonté d'agir comme sujets à part entière et méritent absolument tous les acquis qui résultent de l'action politique et civique. Nous espérons pouvoir contribuer à la normalisation de leur présence dans l'arène politique, en identifiant l'étendue de leur action au Royaume-Uni et en France, ainsi que les modalités de leur engagement. Si les analyses féministes des femmes et de la politique dans les pays occidentaux ont commencé à combler les lacunes laissées par la science politique masculine en documentant les comportements électoraux des femmes et leur participation dans les structures et les processus politiques institutionnels ${ }^{(2)}$, nous en sommes à ce stade de la recherche pour les femmes des communautés musulmanes.

Un deuxième objectif vise à découvrir les sujets qui sont le plus à même de mobiliser les femmes des communautés musulmanes et de les pousser à l'action politique. Comprendre les questions qui sont importantes pour elles permettra non seulement de développer des stratégies qui favorisent leur action politique et leur participation, mais sera utile également pour informer les décideurs politiques sur leurs préoccupations, leur point de vue et leur comportement politique. Plutôt que d'être stigmatisés, les besoins et les aspirations des femmes des communautés musulmanes qui font partie des populations les plus désavantagées socialement et économiquement, en France et au Royaume-Uni, pourraient ainsi être pris en compte par les politiques publiques, qu'il s'agisse des droits politiques ou civiques, de l'immigration et de la nationalité, de la santé, des programmes sociaux ou de l'éducation. Il ne faut pas oublier que, même si la place des femmes a progressé dans les lieux et positions de pouvoir, y compris pour les femmes de minorités ethniques et de groupes religieux, la politique est toujours mise en ceuvre dans des institutions politiques dominées par des hommes. Les Parlements au Royaume-Uni et en France comprennent respectivement 78 \% et $75 \%$ d'hommes $^{(3)}$ qui, encore aujourd'hui, discutent de questions comme le trafic sexuel et la prostitution, décident des conditions régissant une interruption de grossesse et statuent sur le port du voile (hijab) ou de la burqa par les femmes.

\section{Le cadre de l'enquête}

Nous avons utilisé un certain nombre de méthodes de recherches pour ce projet. Nos méthodes comprennent l'intervention sociologique, des entretiens avec des femmes ordinaires et d'autres en position de leadership, l'observation directe au sein d'associations et de réunions publiques impliquant des femmes des communautés musulmanes. 
De plus, nous avons utilisé un questionnaire d'enquête et c'est celui-ci qui sera commenté dans cet article.

Cette enquête est basée sur un sondage national annuel effectué en Grande-Bretagne par l'institut de sondage IPSOS-MORI au nom de la Commission électorale ${ }^{(4)}$ (jusqu'à 2007) et de la Hansard Society ${ }^{(5)}$. IPSOS-MORI nous a accordé la permission d'utiliser son sondage ${ }^{(6)}$ auquel nous avons ajouté une section préliminaire sur le milieu socioéconomique de ces femmes. Notre sondage a eu lieu en décembre 2007 en Grande-Bretagne et en février-mars 2008 en France.

Sur les 120 questionnaires qui étaient prévus dans chaque pays, nous en avons obtenu 119 en Grande-Bretagne et 107 en France. Nous avons sélectionné les sites en fonction de l'âge, du pays d'origine (pakistanaise, bangladeshie, indienne, algérienne, turque, etc.), du statut socio-économique et de la branche de l'islam (par exemple sunnite ou chiite) Les sites sélectionnés comprennent des locaux d'associations, des mosquées et d'autres espaces publics dans les quartiers, comme les centres de formation pour adultes. Une information initiale sur l'enquête a circulé grâce aux associations communautaires, aux mosquées et à des personnalités éminentes. Le sondage a été mené face à face, sur une période de quatre à six semaines. La taille de l'échantillon étant restreinte, les résultats restent fragiles sur certaines questions (il aurait fallu un échantillon de 1000 personnes pour une validité strictement statistique). Les résultats du sondage nous livrent cependant de bons éléments d'information sur les connaissances politiques, l'activisme et d'autres aspects concernant la participation des femmes des communautés musulmanes.

\section{La comparaison Grande-Bretagne-France}

Au préalable, deux éléments méritent d'être mentionnés. Tout d'abord, en 2005, IPSOS-MORI a effectué aussi une étude sur l'engagement politique parmi les populations des minorités ethniques en Grande-Bretagne, ce qui rend possibles certaines comparaisons avec notre sondage. Deuxièmement, il n'existe pas de données comparables au niveau national pour la France sur la participation politique en général et encore moins sur les populations des minorités ethniques. Donc, strictement parlant, les données françaises sur les femmes des communautés musulmanes peuvent seulement être comparées à notre sondage national et notre enquête britanniques. Il reste cependant possible d'établir une comparaison à partir des résultats d'IPSOSMORI, car ces derniers reflètent les modalités générales de participation dans les démocraties libérales de l'Europe occidentale.

Quels résultats avons-nous donc obtenus? Les 13 questions du questionnaire étaient conçues et groupées, comme dans l'étude IPSOS-MORI, dans le but de fournir des 
données sur les aspects de l'engagement politique suivants : connaissances et intérêt ; action et participation (sur la propension à voter et l'activisme); efficacité et satisfaction perçues à propos du système de gouvernement actuel.

Traditionnellement, les études sur la participation politique, qui ont commencé dans les années soixante dans le cadre d'une discipline de science politique dominée par les hommes, ont en général dépeint une image négative des femmes quant à leur participation à la vie civique et politique. À l'heure actuelle, la conviction que les femmes ont moins de connaissances et d'intérêt en politique persiste toujours malgré les efforts déployés par les politologues féministes, depuis les années soixante-dix, pour proposer une image différente et remettre en question les définitions et les typologies de la participation politique qui empêchent les femmes de s'impliquer, malgré les stratégies développées pour accroître la présence des femmes dans les décisions politiques. Ces préjugés s'accentuent encore quand est considérée la participation politique des femmes venant de milieux désavantagés ou originaires de pays en voie de développement dans lesquels la démocratie libérale n'est pas encore bien établie. En ce qui concerne les femmes venant de communautés musulmanes, les discours publics et populaires véhiculent l'idée qu'elles s'aventurent rarement hors de la maison ou de la famille, et encore moins quand il s'agit de s'impliquer dans l'arène politique. Les résultats de notre sondage sont, par conséquent, instructifs à ce propos.

\section{Connaissances et intérêt pour la politique}

En ce qui concerne les connaissances et l'intérêt pour la politique, plus de la moitié (55\%) de notre échantillon en Angleterre et presque 68 \% de notre échantillon français nous ont dit qu'elles étaient intéressées par la politique. En dépit des différences transnationales potentielles, l'aspect important de ce résultat est que la majorité écrasante de nos sujets exprime un tel intérêt. En comparant cela à l'enquête nationale, on trouve donc que les femmes des communautés musulmanes en France et en Grande-Bretagne déclarent un intérêt moyen ou élevé pour la politique dans une proportion supérieure à celle du sondage fourni par IPSOS-MORI (51\%) en 2007 $7^{(7)}$. En ce qui concerne les connaissances en politique, 52 \% des femmes que nous avons interrogées en Grande-Bretagne et 45 \% en France ont répondu qu'elles s'y connaissaient assez bien ou très bien. Ces chiffres sont supérieurs à celui de $44 \%$ que nous donne l'enquête nationale d'IPSOS-MORI ${ }^{(8)}$.

Quel que soit le véritable niveau de leurs connaissances en politique, un grand nombre des femmes faisant partie de notre échantillon ont exprimé leur intérêt pour la politique et ont démontré suffisamment de confiance en elles pour nous dire qu'elles avaient des connaissances sur cette question. Autrefois, les sondages sur la participation 
politique se fondaient traditionnellement sur des questions type "quiz". Ces questions portaient le plus souvent sur le nom de responsables politiques ou sur les institutions politiques et leurs fonctions. Cette approche intimidait tout particulièrement les femmes qui soit refusaient de participer au sondage, soit obtenaient de mauvais résultats, avec la conclusion qu'elles démontraient peu d'intérêt et de connaissance en politique. Par ailleurs, pendant notre sondage, les enquêtées pouvaient demander des éclaircissements. On peut noter qu'un nombre significatif de femmes ont demandé ce que voulait dire "s'y connaître sur la vie politique". Immédiatement, elles ont été informées que "la politique" n'était pas confinée aux enjeux soi-disant "difficiles" ou "lourds" comme les affaires étrangères ou l'économie, mais que ce terme comprenait aussi des enjeux proches de leurs intérêts immédiats comme la fermeture définitive des aires de jeux et autres services de proximité pour les jeunes ou le développement des parcs de loisirs des environs pour des raisons industrielles. Ainsi, elles étaient bien plus enclines à dire qu'elles étaient intéressées et s'y connaissaient en la matière. Elles ont également souligné leur intérêt pour la vie politique dans les pays dont elles étaient originaires ou d'où étaient originaires leurs parents.

\section{Action et participation}

Le deuxième volet du sondage portait en premier lieu sur la participation politique et plus précisément sur la probabilité de participer aux élections. Les résultats sont les suivants : à propos de la probabilité de voter, 60,5\% des femmes interrogées en Grande-Bretagne nous ont dit qu'elles étaient absolument certaines de voter dans une élection nationale proche, alors que seulement $5 \%$ ont déclaré qu'elles étaient certaines de ne pas voter. Dans l'échantillon français, un pourcentage étonnant de $88 \%$ annonce la certitude de voter lors d'une élection nationale à brève échéance. Ce chiffre, anormalement élevé, s'explique peut-être par le contexte français au moment de l'enquête. Notre sondage français, en février et mars 2008, a suivi de près deux élections françaises âprement disputées (présidentielle et parlementaire). Par ailleurs, l'élection présidentielle a été le témoin de la défaite du candidat féminin du parti socialiste et de l'ascension à la présidence de Nicolas Sarkozy qui s'était fortement aliéné les populations immigrées, les minorités ethniques et les communautés musulmanes en France. Il avait aussi été confronté à des manifestations de masse en 2007 contre les réductions de financement dans le secteur public et contre la réforme des retraites. Quoi qu'il en soit, les chiffres, français et britanniques, sont tous deux élevés si on les compare aux chiffres de l'étude nationale selon laquelle $53 \%$ de la population britannique étaient certains de voter si une élection devait avoir lieu au moment de l'enquête. 
Un autre aspect de l'engagement politique examiné est celui de l'action politique et notamment du type de participation : une question sur les types de participation considérés comme plus "conventionnels", avec une liste de 12 activités qui comprennent, entre autres, "participer à des élections", "briguer une position d'élu", "écrire une lettre à un journal" ou "contacter un élu". La participation moins conventionnelle à la politique est examinée à partir d'une liste de 17 activités, comme boycotter certains produits ou contribuer à financer un parti politique. Sur ce plan, les résultats de notre échantillon britannique indiquent que les femmes des communautés musulmanes sont aussi actives politiquement, sinon plus, que les femmes qui figurent dans l'étude IPSOS-

\section{En France, le profil de la représentation des minorités ethniques, et donc des musulmans, est beaucoup moins positif au niveau national, bien que plus encourageante au niveau municipal.} MORI. Par exemple, $32 \%$ des femmes de notre échantillon britannique et 12,5 \% de l'échantillon français affirment qu'elles ont contacté un élu (soit un conseiller municipal soit un député), tandis que seulement $15 \%$ de l'échantillon de l'étude nationale déclarent l'avoir fait. De plus, 6,7 \% de notre échantillon britannique et 1,3\% de l'échantillon français signalent avoir versé un don à caractère politique (4\% dans l'échantillon de l'étude nationale).

Les différences entre les chiffres britanniques et français peuvent en partie s'expliquer par le fait que les femmes de culture musulmane en Grande-Bretagne sont impliquées dans le système politique à plus grande échelle que leurs homologues en France et sont, de fait, plus susceptibles de contacter un représentant politique. Cet argument peut être appuyé par une référence à la représentation politique des minorités ethniques et des musulmans en Grande-Bretagne et en France. En Grande-Bretagne, la représentation des minorités ethniques à la Chambre des communes s'élève à $4 \%$, par rapport à une représentation des minorités ethniques estimée à $12 \%$ de la population dans son ensemble. Sur les 28 parlementaires issus des minorités ethniques, 11 sont des femmes. Quant à leur appartenance religieuse, 3 sont des femmes musulmanes et 6 sont des hommes musulmans ${ }^{(9)}$. Au niveau des assemblées municipales, 4 \% des conseillers locaux sont issus des minorités ethniques, bien que les femmes issues de minorités ethniques ne représentent que $1 \%$ du nombre total des conseillers britanniques ${ }^{(10)}$. Les chiffres concernant les conseillers musulmans de sexe féminin sont actuellement indisponibles. En France, le profil de la représentation des minorités ethniques, et donc des musulmans, est beaucoup moins positif au niveau national, bien que plus encourageante au niveau municipal. De fait, les chiffres de l'Assemblée nationale et du Sénat montrent que la représentation des minorités ethniques est insignifiante, soit moins de $1 \%$ pour les deux chambres réunies ${ }^{(11)}$. Pour autant, au niveau municipal, 
on estime que presque $7 \%$ des candidats élus sont issus des minorités ethniques ${ }^{(12)}$ par rapport à une estimation de $12,6 \%$ pour la population générale ${ }^{(13)}$. Or il est collectivement admis que c'est la présence des minorités ethniques dans les politiques nationales qui promeut le plus l'intérêt pour l'engagement dans le système politique de la part des populations issues des minorités ethniques.

\section{Perception de l'efficacité et niveau de satisfaction vis-à-vis de l'action gouvernementale}

Finalement, et très brièvement, en ce qui concerne le troisième aspect de la participation politique étudiée, à savoir son efficacité dans le fonctionnement du système de gouvernement actuel, notre sondage en France et en Grande-Bretagne suggère que les femmes des communautés musulmanes se rangent dans les catégories qui vont d'assez à très optimistes : 38 \% de femmes en Grande-Bretagne et 40 \% en France pensent pouvoir changer les choses en s'engageant politiquement, contre moins d'un tiers des personnes interrogées dans l'étude IPSOS-MORI. Par ailleurs, la grande majorité des femmes de culture musulmane, contrairement à l'échantillon d'IPSOS-MORI, pensait qu'il restait beaucoup à faire pour améliorer le système de gouvernement actuel.

Ces résultats démontrent que la participation politique des femmes des communautés musulmanes est significative et dépasse souvent celle de la population britannique en général, qu'il s'agisse ou non de méthodes conventionnelles. Les résultats nous indiquent que ces femmes s'impliquent dans différents types de participation, et finalement qu'elles aimeraient avoir davantage d'influence politique et apporter leur concours aux améliorations du système.

\section{Conclusion}

Nous espérons que cette étude contribuera à combler les lacunes de la recherche sur la participation politique des femmes des populations musulmanes et constituera un premier pas pour changer la perception publique sur ces femmes comme acteurs politiques. Un élément tout aussi important de notre recherche, s'il est vrai que la politique détermine qui obtient quoi, où et quand (d'après le titre du livre de Harold Lasswell en $\left.1936^{(14)}\right)$, et si la participation politique est une question d'acquis, c'est de découvrir comment et pourquoi certaines personnes ou certains groupes sociaux réussissent et d'autres pas. Les femmes des communautés musulmanes en GrandeBretagne et en France ne comptent pas parmi ceux qui réussissent. En Grande-Bretagne, elles connaissent le taux le plus élevé d'inactivité économique, elles évoluent au sein 
de populations aux indicateurs de pauvreté les plus élevés, et 43 \% d'entre elles n'ont aucun diplôme ni qualification ${ }^{(15)}$. Bien que nous n'ayons pas de statistiques comparables pour la France, on peut avancer sans trop de risques de se tromper qu'elles sont sujettes à l'exclusion sociale étant donné qu'elles font partie d'une minorité visible et/ou de populations immigrées. Par conséquent, il est important de savoir comment mobiliser davantage cette population de femmes dans les deux pays et de déterminer si, grâce à leurs modes de participation, elles parviennent à convaincre les décideurs politiques de cesser de stigmatiser leur présence dans la société et de commencer à répondre à leurs besoins et à leurs espoirs.

\section{Notes}

1. Étude financée par le Economic and Social Research Council ("Women from Muslim Communities and Politics in Britain and France", ref RES-062-23-0380), entre le 1 juin 2007 et le 31 mai 2011.

2. Gill Allwood and Khursheed Wadia, Women and Politics in France: 1958 -2000, London, Routledge, 2000 ; Françoise Gaspard (dir.), Les Femmes dans la prise de décision en France et en Europe, Paris, L'Harmattan, 1997 ; William Guéraïche, "Women in French Political Life, from the Liberation to the 1970s. Essay on the Distribution of Power", thèse de doctorat, université de Toulouse-Le Mirail, 1992 ; Patricia Latour, Monique Houssin et Tovar Madia, Femmes et citoyennes: du droit de vote à l'exercice du pouvoir, Paris, Les Éditions de l'Atelier, 1995 ; Andrée Michel, "Les Françaises et la politique", in Les Temps Modernes, n² 230, 1965, pp. 61-91 ; Janine Mossuz-Lavau et Mariette Sineau, "France", in Joni Lovenduski and Jill Hills (dir.), The Politics of the Second Electorate. Women and Public Participation, London, Routledge \& Kegan Paul, 1981, pp. 112-133; Janine Mossuz-Lavau et Mariette Sineau, Enquête sur les femmes et la politique en France, Paris, PUF, 1983 ; Mariette Sineau, Des femmes en politique, Paris, Economica, 1988 ; Donna Sanzone, "Women in Positions of Political Leadership in Britain, France and West Germany", in Janet Siltanen and Michelle Stanworth (dir.), Women and the Public Sphere, London, Hutchinson, 1984, pp. 160-175.

3. IPU (Interparliamentary Union), Women in National Parliaments, 30 september 2012. www.ipu.org/wmn-e/classif.htm.

4. La Commission électorale est une organisation indépendante, créée par le Parlement du Royaume-Uni, qui a pour fonction la réglementation du financement des partis politiques et des élections ainsi que la définition des normes pour les élections.

5. "Hansard" est le nom donné aux transcriptions officielles des débats parlementaires au Royaume-Uni. La Hansard Society est un organisme de recherche et d'enseignement politique, indépendant de toute institution gouvernementale et parlementaire.

6. Notre enquête est fondée sur le deuxième sondage national portant sur l'engagement politique, effectué en 2004 et comprenant 13 questions (Electoral Commission and Hansard Society 2005).

7. Hansard Society, Audit of Political Engagement 5. The 2008 Report, London, Hansard Society, 2008, p. 50.

8. Ibid.

9. La représentation des minorités ethniques à la Chambre des lords est légèrement supérieure à 5 \%.

Sur les 42 membres de la Chambre des lords issus des minorités ethniques, 10 sont musulmans, dont 4 sont des femmes. Richard Cracknell, "Ethnic Minorities in Politics, Government and Public Life", in Social and General Statistics Section, London, House of Commons Library, 2012, pp. 2-6.

10. Ibid., p. 7 .

11. Éric Keslassy, Ouvrir la politique à la diversité, Paris, Institut Montaigne, 2009, pp. 19-20.

12. Haut Conseil à l'intégration (HCI), Études et intégration. Faire connaître les valeurs de la République. Les élus issus de l'immigration dans les conseils municipaux (2001-2008), rapport au Premier ministre, Paris, La Documentation Française, 2010, p. 115.

13. Richard Cracknell, "Ethnic Minorities in Politics, Government and Public Life", op. cit., p. 6.

14. Harold D. Lasswell, Politics: Who Gets What,When, How, San Francisco, Whittlesey House, 1936.

15. Office of National Statistics (ONS), "Sex and Age and Socio-Economic Classification by Religion", 2003. www.ons.gov.uk/ons/.../commissioned-output-table-finder.xls. 